\title{
Medición de la pobreza multidimensional en América Latina a través de modelos estructurales
}

\author{
Henry Laverde-Rojas*, John Jairo Gómez-Ríos**
}

* Doctorando en Economía. Profesorinvestigador, Facultad de Economía, Universidad Autónoma de Colombia, Bogotá, D.C.

Correo electrónico: hlaverde@gmail.com

** Magister en Economía. Profesorinvestigador, Facultad de Economía, Universidad Autónoma de Colombia, Bogotá, D.C.

Correo electrónico:

johgome@gmail.com

Recibido: 14 de marzo del 2015

Aprobado: 30 de abril del 2015

Cómo citar este artículo: Laverde-Rojas, H. y Gómez-Ríos, J. J. (2015). Medición de la pobreza multidimensional en América Latina a través de modelos estructurales. Cooperativismo \& Desarrollo, 23(106), 89-110. doi: http://dx.doi.org/10.16925/ co.v23i106.1130

\section{Resumen}

Introducción: el presente artículo estima un índice de pobreza multidimensional mediante un método que permite obtener las ponderaciones que se asocian a indicadores utilizados. En la literatura, generalmente, para calcular este tipo de medidas compuestas, se asignan valores arbitrarios a dichas ponderaciones. Por lo tanto, esta manera de operar deja por fuera las subjetividades presentes que pueden darse en los cómputos al establecer arbitrariamente los pesos, evitando los sesgos de estimación. Metodología: la metodología utilizada es conocida como mínimos cuadrados parciales en modelos de ruta (PLS-PM por sus siglas en inglés). El modelo presentado estima las ponderaciones bajo un sistema de ecuaciones que permite observar de manera objetiva la importancia relativa de los indicadores relacionados con la pobreza. Resultados: el aporte fundamental de este artículo es que, a diferencia de otros estudios que emplean enfoques multidimensionales, se consigue estimar de manera sistemática las ponderaciones que cada dimensión aporta a la construcción del indicador de pobreza. Un segundo aporte de este estudio es que obtiene las ponderaciones mediante una novedosa metodología, la cual ha sido poco explorada en áreas de economía. Conclusiones: la aplicación empírica de la metodología propuesta permite dilucidar las grandes diferencias entre dos países latinoamericanos. Esto revela los marcados contrastes entre estos dos países a pesar de estar en regiones relativamente cercanas.

Palabras clave: modelos estructurales, PLS-PM, pobreza multidimensional. 


\title{
Measurement of Multidimensional Poverty in Latin America Using Structural Models
}

\begin{abstract}
Introduction: This article estimates the multidimensional poverty index using a method to obtain weightings associated with the indicators used. Generally in the literature, to calculate this type of compound matters, arbitrary values are assigned to those weightings. This way of operating therefore leaves aside the subjectivities present that could arise in computations when arbitrarily establishing the weights, thus avoiding biases of estimation. Methodology: The methodology used is known as the partial least squares regression in path modeling (PLs$\mathrm{PM}$ ). The model estimates the weightings under a system of equations that makes it possible to objectively observe the relative importance of the indicators associated with poverty. Results: The fundamental contribution of this article is that, in contrast to other studies that use multidimensional approaches, it is able to systematically estimate the weightings that each dimension provides in building the poverty indicator. A second contribution from this study is that the weightings are obtained using an innovative methodology that has been little explored in areas of economics. Conclusions: The empirical application of the proposed methodology makes it possible to elucidate the great differences among two Latin American countries. This shows the market contrasts among these two countries despite their location in relatively nearby regions.
\end{abstract}

Keywords: structural models, PLS-PM, multidimensional poverty.

\section{Medição da pobreza multidimensional na América Latina por meio de modelos estruturais}

\section{Resumo}

Introdução: este artigo estima um índice de pobreza multidimensional mediante um método que permite obter as ponderações que são associadas com indicadores utilizados. Na literatura, geralmente, para calcular esse tipo de medidas compostas, designam-se valores arbitrários a essas ponderações. Portanto, essa maneira de operar deixa de fora as subjetividades presentes que podem ocorrer nos cômputos ao estabelecer arbitrariamente os pesos, o que evita as distorções de estimativa. Metodologia: a metodologia usada é conhecida como Quadrados Mínimos Parciais em modelos de rota (PLS-PM, por sua sigla em inglês). O modelo apresentado estima as ponderações sob um sistema de equações que permite observar de maneira objetiva a importância relativa dos indicadores que se referem à pobreza. Resultados: a contribuição fundamental deste artigo se deve a que, à diferença de outros estudos que empregam enfoques multidimensionais, se consegue estimar de maneira sistemática as ponderações que cada dimensão contribui para a construção do indicador de pobreza. Uma segunda contribuição deste estudo é a obtenção das ponderações mediante uma nova metodologia, a qual tem sido pouco explorada em áreas da economia. Conclusões: a aplicação empírica da metodologia proposta permite esclarecer as grandes diferenças entre dois países latino-americanos. Isso revela os marcados contrastes entre esses dois países apesar de estarem em regiões próximas.

Palavras-chave: modelos estruturais, PLS-PM, pobreza multidimensional. 


\section{Introducción}

La pobreza es un tema de vital importancia para América Latina, ya que es uno de los principales fenómenos sin resolver para nuestra sociedad. Resulta poco comprensible que actualmente existan grupos de la población excluidos y que sufran situaciones de pobreza extrema. Es fundamental investigar sobre aquellos aspectos que afectan el bienestar de los individuos, en especial si se generan situaciones de desarraigo social. Por otra parte, resulta paradójico que, siendo la economía una ciencia que se encarga de la mejor administración de los recursos escasos, no haya podido encontrar una solución definitiva a este fenómeno.

Por esta razón, la presente investigación pretende brindar un aporte a la comunidad científica que se encuentra interesada en encontrar una forma sencilla de medir la pobreza en Colombia y Chile y de explicar sus causas, con el fin de actuar sobre ella a través de una metodología novedosa que puede brindar mayores aportes que los realizados hasta el momento. De esta manera, la delimitación se centra en un progreso de la visión tradicional de la pobreza hacia un estado multidimensional, y acotando el análisis empírico a la situación colombiana y chilena.

Los modelos de ecuaciones estructurales resultan ser un método valioso para medir la pobreza multidimensional ya que a través de ellos se pueden establecer relaciones de causalidad entre las dimensiones que esta incluye y las variables que se toman en cuenta en estas. Así mismo, también se puede establecer la importancia relativa de cada una de estas dimensiones y a su vez de cada una de las variables mediante la estimación estadística de ponderadores, conjugada con elementos teóricos que le brindan un soporte formal a los resultados.

Basados en información proveniente de la Encuesta Nacional de Calidad de Vida (ENCv) para Colombia en el 2012 y la Encuesta de Caracterización Socioeconómica Nacional (Casen) para el caso de Chile en el 2009, la presente investigación elabora un cálculo de la pobreza multidimensional (PM) a través un modelo de ecuaciones estructurales. Uno de los aspectos importantes a tener en cuenta en la presente investigación consiste en la elección de la unidad de análisis para medir la pobreza, la cual tradicionalmente se ha cuantificado a través del ingreso individual o del hogar. Esta visión puede generar resultados sesgados ya que no existe una combinación de elementos objetivos y subjetivos ni se tienen en cuenta aspectos claves que también influyen en dicha medición tales como el trabajo, la vivienda, el ocio, entre otros. Por tal razón, la unidad de análisis para medir la pobreza será el ingreso per cápita.

Por su parte, es importante tener en cuenta el criterio por el cual se eligieron estos países. Colombia y Chile comparten varios elementos en común. En esencia, ambos países pertenecen a América Latina, lo cual los relaciona en varios aspectos culturales, tales como el idioma, la religión y los ancestros indígenas, entre otros. No obstante, en términos económicos, Chile es considerado como un país líder en la región dado el alto nivel de vida de su población y el óptimo desempeño económico. Según datos del Foro Económico Mundial (2014), el ingreso per cápita de Chile es de us $\$ 15.776$, mientras que el de Colombia es de us $\$ 8.098^{1}$. Esto ubica al país austral en el puesto 45 a nivel global y en el segundo a nivel suramericano, después de Uruguay, mientras que Colombia ocupa la posición 68 en el ámbito mundial y el $6^{\circ}$ suramericano (en América Latina y el Caribe, ocupa el $10^{\circ} \mathrm{lu}-$ gar). Unido a lo anterior, estas cifras ubican a Chile dentro del rango inferior de los países con mayores ingresos a nivel mundial, lo cual es un buen referente para Colombia, considerada como una economía en vías de desarrollo.

El análisis anterior se presenta de manera detallada en la primera sección del artículo. En la segunda sección se hace un análisis de antecedentes, en el cual se definen algunos conceptos básicos en torno a la medición de la pobreza y a la elección de la variable de estudio; también se realiza una revisión de la literatura en cuanto al tema de pobreza y la metodología a utilizar. Posteriormente, se hace una descripción detallada del modelo a emplear y se muestran los principales resultados obtenidos. Por último, se concluye y se plantean algunas recomendaciones.

\section{Antecedentes}

De acuerdo con Poza (2008), se puede decir que un individuo es pobre cuando su nivel de vida en relación con su entorno está por debajo de un determinado umbral que se puede considerar mínimo.

Sin embargo, el concepto de pobreza reviste un entorno abstracto y singular por lo que tiene varias acepciones desde un punto de vista conceptual. En general, basándose en la literatura relevante sobre el

Cifras en dólares corrientes de 2013. 
tema (Atkinson, 1983, 1987; Martínez-Álvarez, 2003) vale la pena distinguir dos definiciones fundamentales, a partir del punto de vista a considerar:

Pobreza objetiva: medida a través de criterios objetivos (hechos) de los hogares o de las personas, esto es, las mediciones se basan en un nivel de vida mínimo que responde a criterios prefijados a priori. Se mide a través de indicadores como los niveles de ingresos y gastos y dotaciones de la vivienda.

Pobreza subjetiva: conocida como pobreza por insatisfacción, la cual define como pobre a quien no está satisfecho con su situación, al estar excluido de lo que el individuo considera como "modo normal" de vida, independientemente de sus posibilidades económicas. En otras palabras, es una propia percepción del individuo o del hogar, donde se ve a sí mismo como pobre.

Además de estos dos conceptos, en varios estudios sobre pobreza se han distinguido términos como la pobreza absoluta, según la cual son pobres quienes carecen de lo necesario para la subsistencia, esto es, aquellas personas que no pueden permitirse cubrir las necesidades consideradas como mínimas. Este concepto se adapta mejor para estudios en países en vías de desarrollo, ya que es allí donde se encuentran problemas reales para obtener la renta mínima para subsistir.

La pobreza relativa, por otra parte, define a un individuo como pobre cuando se encuentra en condiciones de inferioridad de recursos respecto a su entorno, aunque su situación no pueda ser considerada tan apremiante como la anterior. Esto implica que la pobreza relativa incorpora las dimensiones espacio y tiempo para medir tal situación ${ }^{2}$.

Este concepto es generalmente utilizado para medir la pobreza en países desarrollados, ya que está más cerca del concepto de exclusión social. De hecho, la Unión Europea lo emplea dentro de su definición oficial de pobreza.

Además de los conceptos arriba mencionados, la literatura sobre el tema también ha incorporado algunas distinciones tales como (Barr, 1998; Ureña, 1999; López-Aranguren, 2005):

Pobreza estática: refleja la situación de pobreza en un momento determinado del tiempo.

Pobreza dinámica: mide la duración de la pobreza en función del tiempo. Permite establecer la

2 Por ejemplo, no es lo mismo ser pobre en Luxemburgo que serlo en Haití, ni serlo en Luxemburgo hoy que serlo hace treinta años. duración de los individuos en situación de pobreza, así como cuáles son sus trayectorias de entrada y salida. Por medio de este método se pueden averiguar las causas que llevan a los individuos u hogares a caer en situación de pobreza así como las medidas de política pública que pueden permitir su salida.

Esteconcepto, permitedistinguirlapobrezatransitoria y la pobreza permanente, según sea la duración de la situación de escasez de recursos.

Pobreza integral: tiene en cuenta la escasez de ingresos, así como la dificultad de acceso a los servicios sociales que facilitan la cobertura de las necesidades vitales básicas.

Nueva pobreza: tiene en cuenta a los afectados por las transformaciones industriales provocadas por las innovaciones tecnológicas y otras circunstancias sociales o económicas: trabajadores desempleados, subempleados, empleados precarios, trabajadores en la economía sumergida, entre otros. Por lo tanto este concepto está muy relacionado con la precariedad laboral.

Pobreza unidimensional: parte de la perspectiva de una única variable objeto de análisis. Se centra fundamentalmente en el análisis de la renta y es el que tradicionalmente se ha utilizado en los estudios de la economía de la pobreza.

Pobreza multidimensional: estudia el fenómeno de la pobreza desde un enfoque mucho más completo, de ahí que se pueda mezclar con la pobreza integral, ya que incorpora otros factores además del meramente monetario, tal como el estado de la vivienda, salud, educación, empleo y relaciones sociales.

Esta concepción es la más compleja de todas pues incorpora un mayor número de variables explicativas del fenómeno, por tanto, la precisión del mismo mejora, ayudando a describir y explicar de manera más eficiente el fenómeno de la pobreza.

Definición del umbral de pobreza: para el estudio de la pobreza es necesario definir un umbral o línea a partir del cual todos aquellos que se encuentren por debajo sean considerados como pobres; de esta manera se puede trazar una línea imaginaria que separe a los pobres de aquellos que no lo son.

Las líneas de pobreza están asociadas con sus respectivos conceptos, de tal forma que pueden distinguirse, entre otros: umbral de pobreza absoluto, umbral de pobreza relativo, umbral de pobreza subjetivo y umbral de pobreza multidimensional.

Línea de pobreza absoluta: se hace una estimación del costo de una cesta básica de bienes de consumo y se identifica como pobre a todo individuo cuyo 
nivel de renta ajustada no alcance para adquirir dicha cesta.

De acuerdo con Atkinson (1983), se define un vector de necesidades mínimas $\left(X^{*}\right)$ que nos proporcionará una línea de pobreza $U$ :

$$
U=(1+h) P X^{\star}
$$

$P=$ Precios de los bienes definidos.

$h=$ Bienes no incluidos en $X^{*}$, siempre que sean necesarios.

Para que esta formulación tenga validez, los bienes incluidos en $X^{\star}$ deben ser medibles en unidades monetarias. Si $h$ representa bienes no incluidos en la renta $X^{\star}$, puede depender de la media proporcional que los hogares gasten en alimentos.

La principal desventaja en relación con la utilización de este tipo de umbrales de pobreza radica en que no existe un único nivel de subsistencia que pueda ser usado como base para la fijación de una línea de pobreza. Por ejemplo, resulta difícil determinar los requerimientos alimenticios de la población con cierta precisión, ya que su nivel mínimo de consumo depende del nivel de actividad, del trabajo, de la región donde se encuentre, entre otros.

Línea de pobreza relativa: se fija con respecto a algún parámetro de la distribución del ingreso o gasto de la población. En este tipo de líneas suelen tomarse como referencia la media o la mediana de la distribución total; aunque también pueden tomarse como referencia los percentiles.

Como aspecto desfavorable para destacar con respecto a este umbral de pobreza, es importante tener en cuenta que un incremento proporcional del ingreso de la población puede dejar invariante el porcentaje de pobres, con lo que un desarrollo económico proporcionalmente distribuido no reduce los porcentajes de pobreza; aunque sí es cierto que mejora el bienestar en términos absolutos.

Línea de pobreza subjetiva: se determina teniendo en cuenta las apreciaciones subjetivas de cada individuo u hogar sobre sus propias necesidades. Este tipo de línea es muy importante, ya que son calculadas a través de la percepción de la propia población, quienes son los mejores conocedores de su situación. Este tipo de umbral se calcula mediante una encuesta directa a la propia población. En este sentido, Ureña (1999) y Martínez-Álvarez (2003) identifican las metodologías de carácter subjetivo más usuales:
Método de encuesta directa: intenta sintetizar la información (subjetiva) proporcionada por cada individuo u hogar, y aplicarla a toda la población que, a través de la siguiente pregunta, crea un modelo para estimar las respuestas: "En su opinión, ¿cuáles son los ingresos mensuales que como mínimo se necesitan para que un hogar como el suyo llegue a fin de mes?".

Bajo la hipótesis de que el ingreso mínimo que el hogar declara para llegar a fin de mes depende fundamentalmente de su tamaño y del nivel de ingresos que tiene, se construye un modelo que relaciona estas tres variables, en donde la variable dependiente es el ingreso mínimo para llegar a fin de mes y las variables independientes son el tamaño del hogar y el ingreso real.

El modelo consiste en estimar la ecuación siguiente:

$$
\log y^{*}=\beta_{0}+\beta_{1} \log f i+\beta_{2} \log y i+u i
$$

Donde,

$f=$ tamaño del hogar

$y=$ ingreso real

$y^{*}=$ ingreso mínimo subjetivo indicado en la respuesta

La cifra resultante para cada valor de $f$ es el umbral de la pobreza para los hogares de ese tamaño, con lo que todos los que se encuentren por debajo de ella, serán considerados como pobres.

La principal crítica que se ha hecho a esta línea radica en que la manera de formular la pregunta induce a los hogares a sobreestimar sus necesidades mínimas, con lo que sesgará los resultados.

Línea de pobreza de Leyden: se basa en la función de bienestar de la Renta (WFI), que se calcula a partir de una encuesta (Encuesta de evaluación de la renta, IEQ). Para Leyden, es pobre aquel hogar que tiene un ingreso familiar después de impuestos, inferior a un nivel de utilidad medido por la función de bienestar de la renta.

Aunque la metodología de cálculo de esta línea implica ciertas complicaciones, esto se compensa con unos resultados más ajustados, ya que las familias suelen ser bastante más realistas en la evaluación de sus necesidades.

Línea de pobreza multidimensional: bajo este esquema se trata de ampliar la utilización de indicadores más allá de la renta o el gasto y se agregan otros como la educación, el medio ambiente, el ocio o la sanidad. 
La dificultad de estos indicadores mixtos consiste en la medición de variables como el ocio, la sensación de estar cubierto por la sanidad, y a su vez, determinar el nivel mínimo de estas dimensiones.

Actualmente se están utilizando indicadores provenientes de la privación multidimensional, donde se utilizan diferentes bienes y servicios para detectar si los individuos u hogares estudiados manifiestan presencia o ausencia del bien en cuestión.

La variable objeto de estudio. Con respecto a este tema, vale la pena destacar que en la mayoría de los estudios no se tienen en cuenta conceptos como la libertad, los derechos y las oportunidades. En este sentido se presenta un dilema en cuanto a la elección del indicador de bienestar. Así, surgen en la práctica definiciones del nivel de vida en términos de consumo (gasto) o en términos de la capacidad de compra (venta).

Inicialmente, el indicador monetario más adecuado para medir el nivel de vida de un hogar es la suma de los ingresos disponibles de todos sus miembros, aunque en ocasiones estos son subvalorados por los hogares, restándole confiabilidad a los datos. No obstante, para la literatura empírica, a la hora de hacer comparaciones internacionales, la variable renta goza de una mayor homogeneidad en las estadísticas de diferentes países.

Sin embargo, cuanto menor es el período en el que se miden los flujos de renta, mayor será la importancia de sus componentes transitorios y por consiguiente, mayor será la desigualdad estimada y la probabilidad de identificar hogares pobres que lo son de forma muy transitoria. De este modo, el nivel de vida al que se quiere aproximar tiende a ser un fenómeno más estable que la renta anual de los hogares, que con frecuencia varía de un año para otro por razones de tipo coyuntural (Poza, 2008).

De acuerdo con lo anterior, la renta permanente es el concepto que más se ajusta a los objetivos de los estudios de pobreza, ya que se refiere al flujo constante de consumo que una persona podría mantener el resto de su vida, dados su actual nivel de riqueza, sus rentas presentes y sus rentas futuras; todo ello sin que afecte a la fuente de riqueza de la que se derivaría dicho flujo de consumo permanente (Poza, 2008).

Ante la posibilidad de contar con información muestral sobre la renta para períodos superiores a un año, autores como Atkinson (1987) consideran que el gasto (consumo) corriente es un indicador más preciso de la posición a largo plazo que los ingresos corrientes. De esta manera se presenta un consenso desde el punto de vista teórico, según el cual las decisiones anuales de consumo guardan una relación más estable con la renta permanente, de la que existe con los ingresos, ya que estos se ven más influidos por el momento del ciclo vital en el que se encuentran y están más contaminados por los componentes transitorios. No obstante, el gasto está condicionado por los hábitos de consumo y por el ambiente en el que está inmerso el hogar, lo que permite afirmar que no siempre un bajo nivel de consumo está asociado necesariamente a una escasez de medios ${ }^{3}$.

\section{Revisión de literatura}

Dado que la pobreza es un tema de carácter global y es un fenómeno que se presenta en todos los países del mundo, existe una literatura bastante extensa que ha sido enfocada hacia todas las perspectivas posibles en la materia. No obstante, dada su amplia dimensión, la metodología de cálculo, así como las diversas fuentes de información hacen que aún se presenten divergencias en cuanto a los resultados de las estimaciones.

A continuación, se hace una revisión de la literatura reciente en torno al tema y la metodología empleada, teniendo en cuenta las diferentes concepciones que se han adoptado para medir la pobreza en varias de las definiciones arriba planteadas.

El primer documento que vale la pena destacar desde el ámbito internacional, es el de Poza (2008), que, basándose en datos para España desde 1994 hasta 2001 y a través de un análisis factorial confirmatorio de segundo orden, por el método de componentes principales y por rotación no ortogonal, construye un indicador para la pobreza multidimensional. Por otra parte, plantea un modelo explicativo de la pobreza, mediante el método de modelado con ecuaciones estructurales y variables latentes.

Alkire y Santos (2010) buscan un indicador para medir la pobreza extrema. Basadas en datos provenientes de varias fuentes, tales como reportes de desarrollo humano, las autoras realizan un análisis del Índice de Pobreza Multidimensional (IPM) a nivel global mediante una descomposición poblacional por subgrupos de países en vías de desarrollo: Asia del sur, Asia del Este y del Pacífico, África Sub sahariana, América Latina y el Caribe, Estados Árabes y

Por ejemplo, las personas de mayor edad se caracterizan por pautas de consumo más austeras. 
algunos países de Europa Central y del Este. Este documento resulta ser un punto de referencia importante a la hora de realizar investigaciones sobre la pobreza multidimensional, ya que aquí se realiza una descripción acerca de la metodología a emplear para calcular el IPM.

Saboor, Khan, Hussain y Mahmood (2015) examinaron las variaciones regionales y saltos temporales en la pobreza a través de 26 regiones y 4 provincias en Pakistan durante cinco períodos, a saber: 19981999, 2001-2002, 2004-2005, 2005-2006 y 2007-2008, empleando el enfoque de pobreza multidimensional basados en educación, salud e instalaciones de la vivienda. Mediante la metodología estándar desarrollada por Alkire y Foster (2008), reconocida por el Programa de las Naciones Unidas para el Desarrollo (PNUD, 2010), los autores encontraron que la Pobreza Multidimensional (PM) es mayor en las áreas rurales. No obstante, declinó un 7\% en el 2007-2008 frente al periodo 1998-1999 y apenas un 1\% en las áreas urbanas. El estudio sugiere la necesidad de adoptar un enfoque integrado con el ánimo de mejorar las condiciones socioeconómicas de la población y así alcanzar los estándares de bienestar internacionales incorporados en los Objetivos del Milenio 2015.

Para el caso colombiano, la literatura desarrollada en torno a la pobreza ha sido igualmente extensa, destacando en primera medida el documento de Nuñez y Ramírez (2002), que basados en una metodología de descomposición, establecen características de la evolución de la pobreza en la década de los noventa. Como resultado de su investigación concluyen que el incremento de la pobreza entre 1995 y el 2000 se debió totalmente al incremento en el desempleo, aunque también a la inflación y a la mayor regresividad en la distribución del ingreso.

Por su parte, Pérez (2005), con base en los indicadores de necesidades básicas insatisfechas (NBI) y de calidad de vida (ICV), realiza un análisis espacial de la pobreza en Colombia, mediante el cálculo del estadístico I de Moran a nivel local y global. Esta metodología le permite identificar clusters de pobreza y detectar si existe algún proceso de difusión de la pobreza. Sus resultados muestran evidencia de dependencia espacial tanto a nivel departamental como municipal e indican que la ubicación geográfica es importante como determinante de la pobreza en el país. Encontró clusters y procesos de difusión de la pobreza en algunos municipios y departamentos. Se destaca que en la mayoría de los casos los municipios pobres se encuentran rodeados de otros municipios pobres, y que los municipios ricos están rodeados de otros municipios ricos.

López y Núñez (2007) elaboraron un libro que describe y estudia diferentes aspectos de la pobreza en Colombia. Se ocupa, en primera medida, de las diferentes medidas de la pobreza y de las diferentes estimaciones que se hacen en el país; posteriormente analiza los micro y macro determinantes de la pobreza en Colombia; luego se encarga de elaborar un análisis de la magnitud y los efectos del gasto público social sobre la pobreza y la desigualdad y hace un estudio de los instrumentos de focalización utilizados en el país; describe y evalúa el sistema de protección social en Colombia y hace propuestas para su rediseño; finalmente, propone metas a mediano y largo plazo en materia de pobreza y desigualdad y describe las principales estrategias recomendadas: prerequisitos macroeconómicos, educación y capacitación para el trabajo, mercado laboral, pobreza rural, entre otros. Lasso (2008), basado en datos para el período 1998-2007 sobre Colombia, mediante la estimación de índices sociales de precios plutocráticos y democráticos, con base en inflaciones individuales, encuentra evidencia del efecto antipobre de los cambios de precios relativos ocurridos en el periodo en mención y de su regresividad con respecto a la distribución del ingreso. Los resultados muestran que para el 2001, 2002 y 2007, la inflación se tornó regresiva para la distribución del ingreso, lo cual estuvo explicado en los incrementos anuales de los precios de los alimentos, efecto totalmente contrario a lo sucedido en el 2000 y 2003, cuando la inflación de los alimentos estuvo por debajo de la nacional. Igualmente, el autor encontró que incrementos en los precios de los alimentos generan efectos iguales en la inflación nacional, lo cual desencadena aumentos en la pobreza y en la pobreza extrema.

Posteriormente, Angulo, Díaz y Pardo (2011) presentan el índice de pobreza multidimensional (IPM) de Colombia para el periodo 1997-2010, metodología propuesta a partir de cinco dimensiones: 1) condiciones educativas del hogar, 2) condiciones de la niñez y la juventud, 3) salud, 4) trabajo y 5) acceso a los servicios públicos domiciliarios y las condiciones de vivienda. El índice utiliza una estructura de ponderación anidada, en la que cada dimensión tiene el mismo peso y cada variable tiene el mismo peso al interior de cada dimensión. Los resultados de las estimaciones del IPM Colombia muestran que la pobreza multidimensional se redujo en Colombia durante el periodo de análisis. No obstante, persisten grandes desequilibrios entre la población pobre y no pobre. 


\section{Metodología}

Índice de pobreza multidimensional, IPM. La construcción de índices de pobreza multidimensional (IPM) se hace generalmente mediante el agrupamiento de una serie de variables o indicadores que expresan una dimensión diferente del concepto. Los indicadores se expresan de acuerdo a un umbral de privación, los cuales son definidos como la incapacidad que tiene un individuo $i$ de alcanzar un nivel $z_{j}$ en el indicador $x_{j}$, así la privación se da cuando $x_{j}$ $<z_{i}$. Consecuentemente, se debe definir con precisión cuáles serán los criterios para definir los umbrales de privación en cada variable. Una vez que los indicadores han sido seleccionados y los umbrales definidos se debe establecer cuáles serán las ponderaciones que estarán asociadas a cada indicador. Con esto, un valor de privación para cada individuo, $c_{i}$, es encontrado como una suma ponderada del número de privaciones, como se muestra en la ecuación 1 .

$$
c_{i}=w_{1} l_{1}+w_{2} l_{2} \ldots+w_{p} l_{p}
$$

Donde $c_{i} \in[0,1]$, y donde $I_{j}=1$ si el individuo es privado en el indicador j y $I_{j}=0$ en cualquier otro caso, $w_{j}$ es la ponderación atada al indicador $\mathrm{j} \operatorname{con} \sum_{\mathrm{j}=1}^{\mathrm{p}} w_{j}=1$.

Para definir la pobreza multidimensional, se tiene en cuenta un segundo umbral (Foster y Alkire, 2011). Dicho umbral es definido como el porcentaje de privaciones, $k$, que una persona tiene que tener para ser considerada pobre. De esta manera, alguien se considera pobre, si $c_{i} \leq k$. Es común en la literatura considerar pobre a una persona si su valor de privación es igual o más grande que $1 / 3$. Para aquellas personas cuyos valores son menores a este valor, se asigna un valor de 0 , lo que se conoce como censuramiento en la medida de pobreza. Para diferenciar entre los valores originales y los censurados se usa un valor de privación censurado $c_{i}(k)$. Tal notación permite que cuando $c_{i} \geq k$ entonces $c_{i}(k)=c_{i}$, pero cuando $c_{i}<k$ entonces $c_{i}(k)=0$, así, $c_{i}(k)$ es el valor de privación de la pobreza.

Para calcular el IPM se necesitan combinar dos tipos de información. En primer lugar, se debe estimar el porcentaje de personas que experimentaron privaciones múltiples, conocido como índice de recuento multidimensional (н), el cual es calculado como en la ecuación 2.

$$
H=\frac{q}{n}
$$

Donde $q$ es el número de personas que son pobres multidimensionalmente y $n$ es el total de población.

En segundo lugar, debe calcularse lo que se conoce en la literatura como intensidad de la pobreza (A), dado en la ecuación 3.

$$
A=\frac{\sum_{i=1}^{\mathrm{n}} c_{i}(k)}{q}
$$

El valor promedio de privaciones de las personas pobres multidimensionalmente. De esta manera, es posible encontrar el IPM mediante el producto de estas dos medidas, como la ecuación 4 lo muestra.

$$
I P M=H \times A
$$

El problema fundamental de esta metodología se encuentra en las ponderaciones $w_{i}$, pues generalmente se asignan arbitrariamente o ad hoc. En este artículo, por el contrario, estas se consiguen bajo una estimación econométrica. La metodología utilizada es conocida como mínimos cuadrados parciales en modelos de ruta, PLS-PM por sus siglas en inglés. El modelo presentado estima las ponderaciones bajo un sistema de ecuaciones que permite observar de manera objetiva la importancia relativa de los indicadores relacionados con la pobreza. Para la estimación, se emplean cuatro dimensiones de esta: salud, educación, tecnologías de la información y comunicación, condiciones sanitarias e ingresos. De esta manera, se construye un sistema de ecuaciones (5-8) que establecen las relaciones de causalidad entre estos conceptos.

$$
\begin{gathered}
\text { Salud }=\beta_{1}+\beta_{11} \text { Condi }+\zeta_{1} \\
\text { Tecno }=\beta_{2}+\beta_{12} \text { Edu }+\zeta_{2} \\
\text { Edu }=\beta_{3}+\beta_{13} \text { Salud }+\beta_{23} \text { Condi }+\zeta_{3} \\
\text { Ingre }=\beta_{4}+\beta_{14} \text { Salud }+\beta_{24} \text { Edu }+ \\
\beta_{34} \text { Tecno }+\beta_{44} \text { Condi }+\zeta_{4}
\end{gathered}
$$


En este sistema, la salud de los individuos está condicionada por las condiciones sanitarias de los hogares (Condi), la cual se fija como una variable exógena en el modelo. Los aumentos de escolaridad benefician el uso de tecnologías de la información y la comunicación (Tecno). La educación, por su parte, estará condicionada por la salud y por las condiciones sanitarias de los hogares. Finalmente, los ingresos de los individuos dependerán de la salud, educación y las TIC. Aunque estas relaciones de causalidad establecidas pueden ser discutibles, se basan en la literatura alrededor de la pobreza. Sin embargo, para probar la robustez de los resultados se harán algunos análisis de sensibilidad posteriormente, generando diferentes relaciones en el sistema.

Por otro lado, cada una de las dimensiones forma un bloque que contendrá uno o varios indicadores para medirlo. La tabla 1 muestra las diferentes dimensiones con sus respectivos indicadores y los umbrales de privación de cada una de ellos.

En los modelos PLS-PM, cada dimensión es construida por sus indicadores, de esta manera existen dos formas de hacerlo:

- Modo A o reflectivo: en este caso cada uno de los indicadores es una consecuencia del mismo concepto. De esta manera, los diferentes indicadores deben covariar. Se espera que si el concepto se mueve hacia una dirección, todos los indicadores los hagan en la misma.

- Modo B o formativo: esta manera de estimación supone que los conceptos son el resultado combinado de los indicadores.

En la literatura es frecuente utilizar el modo reflectivo, asegurándose de hacer una transformación a las variables de tal manera que estén correlacionadas positivamente; este será el caso de este estudio. Tal elección debe asegurar que los diferentes indicadores midan lo que tratan de caracterizar, es decir que sean unidimensionales. Para ello, se cuenta con una batería de test que aseguran la unidimensionalidad de los indicadores. De esta manera, los parámetros del modelo son obtenidos mediante una regresión simple de los indicadores sobre su concepto subyacente $\vartheta$ por MCO, como se muestra en la ecuación 9.

$$
X_{q}=\vartheta_{q} \lambda_{q}^{T}+\epsilon_{q} \quad E\left(\epsilon_{q} / \vartheta_{q}\right)=0
$$

Tabla 1

Descripción de dimensiones y variables

\begin{tabular}{|c|c|c|}
\hline Dimensión & Indicador & $\begin{array}{l}\text { Criterio para definir } \\
\text { umbrales de privación }\end{array}$ \\
\hline Ingreso & $\begin{array}{l}\text { Ingreso per cápita de } \\
\text { los hogares }(\mathrm{Y})\end{array}$ & 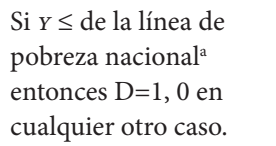 \\
\hline \multirow{3}{*}{ Educación } & $\begin{array}{l}\text { Años promedios de } \\
\text { educación (APE) }\end{array}$ & $\begin{array}{l}\text { Si } A P E \leq 6 \text { entonces } \\
D=1,0 \text { en cualquier } \\
\text { otro caso. }\end{array}$ \\
\hline & $\begin{array}{l}\text { Analfabetismo } \\
\text { (ANA) }\end{array}$ & $\begin{array}{l}\text { Si no sabe leer y escri- } \\
\text { bir entonces } D=1,0 \text { en } \\
\text { cualquier otro caso. }\end{array}$ \\
\hline & $\begin{array}{l}\text { Educación de los } \\
\text { padres (EDUPA) }\end{array}$ & $\begin{array}{l}\text { Si } E D U P A \leq 6 \text { entonces } \\
\mathrm{D}=1,0 \text { en cualquier } \\
\text { otro caso. }\end{array}$ \\
\hline \multirow{3}{*}{ Salud } & $\begin{array}{l}\text { Afiliación a sistemas } \\
\text { de salud (AFI) }\end{array}$ & $\begin{array}{l}\text { Si no está afiliado a un } \\
\text { sistema de salud enton- } \\
\text { ces } D=1,0 \text { en cualquier } \\
\text { otro caso. }\end{array}$ \\
\hline & $\begin{array}{l}\text { Estatus de salud } \\
\text { (sALU) }\end{array}$ & $\begin{array}{l}\text { Si el estatus de salud } \\
\text { es menos que regular } \\
\text { entonces } D=1,0 \text { en } \\
\text { cualquier otro caso. }\end{array}$ \\
\hline & Enfermedad (ENFE) & $\begin{array}{l}\text { Si el individuo ha } \\
\text { sido atendido por } \\
\text { enfermedad en hospital } \\
\text { entonces } \mathrm{D}=1,0 \text { en } \\
\text { cualquier otro caso. }\end{array}$ \\
\hline \multirow{3}{*}{$\begin{array}{l}\text { Tecnologías } \\
\text { de la informa- } \\
\text { ción y la } \\
\text { comunicación }\end{array}$} & $\begin{array}{l}\text { Conexión a internet } \\
\text { (INTER) }\end{array}$ & $\begin{array}{l}\text { Si no existe conexión a } \\
\text { internet entonces } D=1 \text {, } \\
0 \text { en cualquier otro } \\
\text { caso. }\end{array}$ \\
\hline & $\begin{array}{l}\text { Uso de telefonía } \\
\text { celular (CELU) }\end{array}$ & $\begin{array}{l}\text { Si el individuo no usa } \\
\text { telefonía celular enton- } \\
\text { ces } D=1,0 \text { en cualquier } \\
\text { otro caso. }\end{array}$ \\
\hline & $\begin{array}{l}\text { Uso de procesadores } \\
\text { (PC) }\end{array}$ & $\begin{array}{l}\text { Si el individuo no usa } \\
\text { procesadores entonces } \\
D=1,0 \text { en cualquier } \\
\text { otro caso. }\end{array}$ \\
\hline $\begin{array}{l}\text { Estándar } \\
\text { de vida }\end{array}$ & $\begin{array}{l}\text { Conexión a servicios } \\
\text { básicos (SERVI) }\end{array}$ & $\begin{array}{l}\text { Si el hogar cuenta con } \\
\text { servicios sanitarios } \\
\text { básicos entonces } D=1 \text {, } \\
0 \text { en cualquier otro } \\
\text { caso. }\end{array}$ \\
\hline
\end{tabular}

Nota. ${ }^{\text {a }}$ Esta línea es calculada con base en el $60 \%$ de la mediana del ingreso per cápita de la muestra seleccionada. Elaboración propia.

Donde los coeficientes $\lambda_{p q}$ son llamados cargas, los cuales asocian la $p$-ésima variable manifiesta con su respectivo concepto en el $q$-ésimo bloque y $\epsilon_{p q}$ es el término de error. 
El modelo estima los parámetros del sistema de ecuaciones (conocido como modelo estructural) y las cargas en los modelos de medida mediante un algoritmo, que resuelve cada modelo de manera parcial. En primera instancia, PLS-PM hace una primera aproximación mediante una combinación lineal de cada concepto con sus propios indicadores, como en la ecuación 10.

$$
\vartheta_{q}^{\prime}=\sum_{j=1}^{p} \alpha_{p} x_{p}
$$

Donde $\vartheta_{q}$ es el concepto subyacente y $\alpha_{p}$ una ponderación arbitraria. Con esta aproximación para cada uno de los conceptos se retorna al sistema de las ecuaciones 5-8. En esta etapa, cada concepto es calculado nuevamente, pero esta vez en relación con los conceptos que lo condicionan (ecuación 11).

$$
\vartheta_{q}^{\prime \prime}=\sum_{q=1}^{Q} \beta_{q} \vartheta_{q^{\prime}}^{\prime}
$$

Con esta nueva estimación, se regresa a la ecuación 9 para estimar las cargas del modelo reflectivo. En esta etapa, se verifica si cumple la siguiente condición de convergencia $\left|\lambda^{\prime}-\alpha_{p}\right|<10^{-5}$, sino el proceso se repite hasta que se alcance. Una vez lograda se realiza una regresión múltiple por mco de cada una de las ecuaciones 5-8. Las propiedades estadísticas de este método son mostradas por Lohmoller (1989). Para los propósitos de este trabajo la metodología permite obtener las ponderaciones de la ecuación 1 mediante una estructura metódica, dejando de lado las arbitrariedades y subjetividades de muchas de las aplicaciones para estimar estas y así los indicadores de pobreza multidimensional. En efecto, el modelo PLS-PM permite estimar la contribución que cada variable aporta a la varianza explicada de cada concepto (a través de las cargas y coeficientes del sistema 5-8). Para hacerlo, primero el bloque de ingresos es tomado como referencia, para luego calcular la contribución que los demás conceptos aportan a este (esto se hace mediante el producto de los coeficientes de cada concepto y la correlación con el bloque de ingresos). Después se relativiza esta contribución en relación con el coeficiente de determinación $R^{2}$ de este bloque. Dicha razón se convierte en las ponderaciones $w_{j}$ utilizadas en la ecuación 1. Es valioso notar que estas ponderaciones son estimadas no solamente en función de su peso relativo entre el bloque de ingreso, sino de una situación de equilibrio, pues se tiene en cuenta las retroalimentaciones que se producen en el sistema de las ecuaciones 5-8.

\section{Datos}

Este artículo presenta un caso de aplicación de la metodología previamente explicada a dos países latinoamericanos: Chile y Colombia. Como se mencionó, la selección de los datos se basó en la literatura disponible, pero además en el cumplimiento de dos criterios. Primero, que en cada país los datos provengan de la misma fuente. Segundo, con el ánimo de comparar los resultados entre estos países se debe hacer uso de variables con equivalencia entre las dos fuentes de datos. Para tal fin, en el caso de Chile se seleccionó la Encuesta Casen, o Encuesta de Caracterización Socioeconómica Nacional, la cual es una encuesta con cobertura nacional, que realiza el gobierno de Chile. Se tomó como referencia el 2009. Para Colombia, se empleó la Encuesta Nacional de Calidad de Vida del año 2012 que realiza el DANE.

Para la estimación del modelo PLS-PM las variables fueron transformadas para que estas estén correlacionadas positivamente, estandarizadas y, para la estimación de los modelos reflectivos, se emplea la técnica centroide. El principal problema en la base de datos se encuentra en los ingresos reportados por los individuos. Una porción importante de estos datos no son reportados, por lo cual, para evitar sesgos en las estimaciones se sigue de cerca la metodología de Gasparini, Gutiérrez y Tornarolli (2007) para hacer imputaciones sobre los datos faltantes. La estimación se realiza por MCO en función de asignarle a un individuo el ingreso de una persona con las mismas características. Una vez conseguidas las ponderaciones son asignadas a cada uno de los indicadores para estimar el IPM, cuyos umbrales de privación son definidos en la tabla 2.

\section{Análisis de resultados}

Como se mencionó, la aplicación de la metodología de la sección precedente para la consecución del 
IPM se realiza para dos países latinoamericanos con dos diferentes niveles de ingresos per cápita: Chile y Colombia. En las últimas décadas, Chile ha tenido un comportamiento destacado en los diferentes indicadores de desarrollo, particularmente este buen rendimiento le ha permitido ingresar a la parte inferior de la distribución de los países de ingresos altos. Colombia, por su parte, ha tenido resultados mixtos $\mathrm{y}$, en la actualidad, es un país de ingreso medio. Esto se convierte en una oportunidad, no solo para aplicar esta metodología a países en desarrollo, sino además, dentro de este grupo, hacer comparaciones para dos países con dinámicas diferentes. Para esto se propone, en primera instancia, presentar los resultados para Chile y luego, reportar los de Colombia haciendo comparaciones con los resultados anteriores.

\section{Pobreza multidimensional para Chile}

La estimación del índice de pobreza multidimensional necesita de las ponderaciones que serán aplicadas a cada indicador en la ecuación 1. Sin embargo, para encontrar de manera consistente estas, en este documento se recurre a un método novedoso que se aleja de la literatura tradicional para estimarlas. Este método es capaz de evaluar la importancia relativa de cada uno de los indicadores, dado que las ponderaciones son estimadas bajo el sistema de ecuaciones presentado en la primera sección. En dicho sistema, cada concepto es calculado bajo un marco de equilibrio a partir de la construcción de bloques, cada uno con un conjunto de indicadores los cuales tratan de medir el concepto subyacente. En este documento, cada bloque es medido por el modo A, de modo que, antes de analizar los parámetros del sistema de las ecuaciones 5-8, es necesario garantizar que cada concepto sea medido con confiabilidad y estabilidad.

De manera más precisa, cada variable utilizada en el sistema es un concepto abstracto que es medido por una serie de variables observables y medibles. Una condición necesaria, por lo tanto, es que dichos indicadores estén fuertemente correlacionados con el concepto que tratan de medir. Se trata, entonces, de que las variables que están en un bloque determinado midan lo mismo, es decir que sean unidimensionales o que covarien. Para verificar esta condición, se recurre a dos tests de unidimensionalidad. El primero, conocido como el Rho de Dillon-Goldstein (rho DG), es un test basado en las cargas obtenidas en las estimaciones, el cual nos indica que para que un indicador sea considerado como parte del bloque, es decir que esté midiendo correctamente el concepto subyacente, el valor de este test debe ser superior a 0,7 . En otras palabras, este test no debe ser inferior a este valor para considerar que un indicador está altamente asociado al concepto que se intenta medir. El segundo test (Igo) se basa en los valores propios de la matriz varianza-covarianza. Para este caso, un indicador será considerado unidimensional si el primer valor propio es superior a 1 , mientras que el segundo es menor a este. La tabla 2 reporta los resultados de unidimensional para los diferentes bloques en el caso de Chile. Como se observa, las condiciones de unidimensionalidad se cumplen satisfactoriamente para todos los indicadores, al observar que tanto los valores de rho de Dillon-Goldstein como los valores propios satisfacen los requerimientos señalados. De esta manera, las estimaciones realizadas garantizan para Chile que los conceptos utilizados para medir el IPM son confiables.

Tabla 2

Unidimensionalidad de los indicadores de pobreza - Chile

\begin{tabular}{cccccc}
\hline & Modo & VM & Rho. DG & 1er. Igo. & 2do. Igo. \\
\hline $\begin{array}{c}\text { Estándar } \\
\text { de vida }\end{array}$ & $\mathrm{A}$ & 1 & 1000 & 1,00 & 0,00 \\
Salud & $\mathrm{A}$ & 2 & 0,700 & 1,07 & 0,93 \\
Educación & $\mathrm{A}$ & 3 & 0,746 & 1,50 & 0,91 \\
TIC & $\mathrm{A}$ & 2 & 0,744 & 1,18 & 0,82 \\
Ingresos & $\mathrm{A}$ & 1 & 1000 & 1,00 & 0,00 \\
\hline
\end{tabular}

Nota. Estimaciones basadas en un modelo reflectivo, esquema centroide y variables estandarizadas. Elaboración propia con base en indicadores para Chile, por Casen, 2009.

Una vez que garantizamos que existe unidimensionalidad en los diferentes bloques, es necesario evaluar las cargas de cada indicador. Estas miden el grado de asociación de un indicador con el concepto que tratan de medir. La idea es tener más varianza compartida que ruido. La literatura alrededor de PLS-PM considera que un indicador es confiable si la carga respectiva es mayor a 0,7 , con esto se consigue que más del 50\% de la varianza sea compartida entre el indicador y el concepto. En la práctica, sin embargo, se cree que este criterio es demasiado estricto, más cuando se trata de muestras de datos grandes y sistemas complejos, tal como el presentado en las ecuaciones 5-8. En este sentido, la literatura empírica utiliza como criterio que la carga no puede ser inferior a 0,4 (Gefen, Straub y Boudreau, 2000). Adicionalmente, 
se puede utilizar el índice de comunalidad que calcula la variabilidad promedio de los indicadores como producto de cambios en el concepto que las relaciona. En general, se considera que este valor debería ser igual o mayor a 0,5 .

La tabla 3 muestra los diferentes indicadores, los bloques a los que pertenecen, su peso dentro del bloque, las cargas y el índice de comunalidad. Los resultados señalan que el único indicador que no cumple con los requerimientos para pertenecer a un bloque (en sentido estricto) es la variable de uso de telefonía celular (CELU) la cual pertenece al bloque de tecnologías de la información y la comunicación, un concepto clave en las nuevas dinámicas de las privaciones

Tabla 3

Pesos y cargas del modelo de pobreza - Chile

\begin{tabular}{lclccc}
\hline & vM & Bloque & Pesos & Cargas & Comunalidad \\
\hline 1 & SERVI & $\begin{array}{l}\text { Estándar de } \\
\text { vida }\end{array}$ & 1000 & 1000 & 1000 \\
2 & AFI & Salud & 0,855 & 0,889 & 0,790 \\
3 & SALU & Salud & 0,660 & 0,723 & 0,672 \\
4 & APE & Educación & 0,762 & 0,928 & 0,861 \\
5 & ANA & Educación & 0,632 & 0,739 & 0,685 \\
6 & EDUPA & Educación & 0,713 & 0,836 & 0,815 \\
7 & CELU & TIC & 0,471 & 0,618 & 0,512 \\
8 & PC & TIC & 0,800 & 0,886 & 0,916 \\
9 & Y & Ingresos & 1000 & 1000 & 0,915 \\
\hline
\end{tabular}

Nota. Estimaciones basadas en un modelo reflectivo, esquema centroide y variables estandarizadas. Elaboración propia con base en indicadores para Chile, por Casen, 2009.

Tabla 4

Correlación por bloques para el modelo de pobreza - Chile que determinan el IPM, el valor de su carga está por debajo de 0,7 . Sin embargo, dada la importancia del indicador y que el valor de la carga está por encima de 0,4 ; además de que su valor de comunalidad está por encima de 0,5 , se decidió mantenerlo dentro del bloque. Es bueno señalar que se probó en diferentes modelos, no reportados, con otras variables dentro de los diferentes bloques, pero fueron excluidas al presentar valores bajos en sus cargas y comunalidades, lo cual les restaba parsimonia a los modelos.

Finalmente, se prueba que los bloques como unidad de medición sean lo suficientemente confiables dados sus indicadores. Una manera de hacerlo es que las correlaciones que presenta cada indicador con su bloque sean más altas que las relaciones de estas con los demás bloques. Es decir, si una variable es lo suficientemente confiable para medir un concepto determinado debería estar más correlacionada con su concepto que con otros conceptos conectados a este. La tabla 4 muestra la matriz de correlaciones de los indicadores con los diferentes bloques, corroborando que ningún indicador está más correlacionado que con el bloque que trata de medir.

En resumen, en esta primera parte se evaluaron las relaciones entre los indicadores y su respectivo concepto. Se puede concluir que el modelo presentado en las ecuaciones 5-8 muestra un buen comportamiento: es confiable (a través de los test de unidimensionalidad y de evaluación discriminante) y estable (mediante los test de confiabilidad de ítem, cargas, y comunalidad), con lo cual se pueden validar los resultados que se presentarán en una segunda etapa, en la cual se analizarán las relaciones de causalidad entre conceptos.

\begin{tabular}{lccccccc}
\hline & Nombre & Bloque & Condi. & Salud & Educa. & TIC & Ingre. \\
\hline 1 & SERVI & Estándar de vida & 1000 & 0,339 & 0,129 & 0,254 & 0,535 \\
2 & AFI & Salud & 0,443 & 0,889 & 0,503 & 0,248 & 0,378 \\
3 & SALU & Salud & 0,504 & 0,723 & 0,468 & 0,245 & 0,455 \\
4 & APE & Educación & 0,637 & 0,670 & 0,928 & 0,515 & 0,678 \\
5 & ANA & Educación & 0,549 & 0,570 & 0,739 & 0,427 & 0,459 \\
6 & EDUPA & Educación & 0,340 & 0,493 & 0,836 & 0,381 & 0,596 \\
7 & CELU & TIC & 0,122 & 0,384 & 0,228 & 0,618 & 0,271 \\
8 & PC & TIC & 0,280 & 0,492 & 0,369 & 0,886 & 0,239 \\
9 & Y & Ingresos & 0,535 & 0,578 & 0,679 & 0,544 & 1000 \\
\hline
\end{tabular}

Nota. Estimaciones basadas en un modelo reflectivo, esquema centroide y variables estandarizadas. Elaboración propia con base en indicadores para Chile, por Casen, 2009. 
En esta segunda etapa se evalúan los coeficientes del sistema de ecuaciones presentado en 5-8. La tabla 5 reporta los resultados de las estimaciones. Los coeficientes, conocidos en la literatura como coeficientes de ruta, muestran las relaciones de causalidad entre las diferentes variables del sistema, en este caso como el modelo fue estimado con variables estandarizadas, cada coeficiente es interpretado como el cambio en desviaciones estándar del concepto dependiente como consecuencia de un cambio en una desviación estándar del concepto relacionado con este. La tabla 5 también muestra el coeficiente de determinación, el cual exhibe la varianza explicada por las variables conectadas a un bloque determinado, así como los errores estándar de los coeficientes de ruta. Un punto central en las estimaciones del modelo PLSPM es que permite obtener no solamente los efectos directos, sino también las retroalimentaciones que se producen entre conceptos de manera indirecta. De esta manera podemos encontrar un efecto total que recoge los efectos directos e indirectos causados por las variables exógenas dentro de un bloque. Dado que PLS-PM no descansa sobre ningún supuesto distribucional, en la base de datos es necesario validar los efectos totales mediante un método de remuestreo, la técnica utilizada fue bootstrapping en la cual se utilizaron 500 remuestras para la validación de los coeficientes. Los resultados señalan que, en promedio, los hogares con mejores condiciones de salud tendrán mejores resultados en los estándares de vida. Este punto puede ser explicado por un efecto residual sobre otras variables.

Las personas más sanas tienden a mejorar tanto sus condiciones productivas como su rendimiento académico, estos hechos sumados inciden sobre su poder de negociación en el mercado laboral reflejándose

Tabla 5

Modelo PLS-PM - Chile

\begin{tabular}{|c|c|c|c|c|}
\hline Bloques & Coeficiente & Directos & Indirectos & Totales \\
\hline \multirow[t]{2}{*}{ Salud $\rightarrow$ Están. } & $0,390^{* * *}$ & 0,390 & & $0,390 \dagger$ \\
\hline & $(0,010)$ & & & $(0,009)$ \\
\hline Coeficiente de determinación, $R^{2}$ & 0,352 & & & \\
\hline \multirow[t]{2}{*}{ Están. $\rightarrow$ Edu. } & $0,119^{* * *}$ & 0,119 & & $0,119 \dagger$ \\
\hline & $(0,009)$ & & & $(0,010)$ \\
\hline \multirow[t]{2}{*}{ Salud $\rightarrow$ Edu. } & $0,247^{* * *}$ & 0,247 & 0,232 & $0,479 \dagger$ \\
\hline & $(0,009)$ & & & $(0,009)$ \\
\hline Coeficiente de determinación, $R^{2}$ & 0,777 & & & \\
\hline \multirow[t]{2}{*}{ Edu. $\rightarrow$ Tecno. } & $0,402^{\star * *}$ & 0,402 & & $0,402 \dagger$ \\
\hline & $(0,009)$ & & & $(0,008)$ \\
\hline Coeficiente de determinación, $R^{2}$ & 0,717 & & & \\
\hline \multirow[t]{2}{*}{ Están. $\rightarrow$ Ingre } & 0,211 & 0,211 & 0,123 & $0,334 \dagger$ \\
\hline & $(0,009)$ & & & $(0,010)$ \\
\hline \multirow[t]{2}{*}{ Salud $\rightarrow$ Ingre } & $0,635^{\star * *}$ & 0,635 & 0,235 & $0,870 \dagger$ \\
\hline & $(0,010)$ & & & $(0,011)$ \\
\hline \multirow[t]{2}{*}{ Edu. $\rightarrow$ Ingre } & $0,515^{\star * *}$ & 0,515 & 0,323 & $0,838 \dagger$ \\
\hline & $(0,011)$ & & & $(0,010)$ \\
\hline \multirow[t]{2}{*}{ Tecno. $\rightarrow$ Ingre } & $0,412^{\star * *}$ & 0,412 & & $0,412 \dagger$ \\
\hline & $(0,011)$ & & & $(0,011)$ \\
\hline Coeficiente de determinación, $R^{2}$ & 0,669 & & & \\
\hline GoF & 0,798 & & & \\
\hline
\end{tabular}

Nota. Modelo estimado para variables latentes estandarizadas. Errores estándar en paréntesis. ${ }^{* *} \mathrm{p}<0,01 .{ }^{\dagger}$ La validación de la significancia de los coeficientes de los efectos totales está basada en intervalos de confianza al 95\% con el método de remuestreo por bootstrapping. Elaboración propia con base en indicadores para Chile, por Casen, 2009. 
esto en las remuneraciones salariales. Estas mejores condiciones económicas impactan por lo tanto sobre la mejora en las condiciones de estándar de vida de los hogares. El impacto que tiene la salud es positivo y altamente significativo, así como la varianza explica esta variable.

Por otro lado, los resultados también señalan que, en promedio, los mejores estándares de vida y de salud también inciden positivamente sobre los rendimientos educativos. Puede decirse que las condiciones más propicias de los hogares (los recursos disponibles, las comodidades, la estructura física, etc.) generan aumentos significativos en la formación de capital humano. Del mismo modo, la salud de los individuos es un elemento clave en dicha formación: los estudiantes más sanos reportan mejores resultados, proyectan su ciclo de vida con retornos más altos, tienen una mayor probabilidad para escolarizarse, etc. De tal manera que estos dos indicadores impactan de manera positiva y fuerte dentro de los logros educativos.

La educación, por su parte, da las condiciones para un mejor aprovechamiento en el uso de tecnologías de la información y la comunicación. En el modelo esta relación se muestra fuerte y altamente significativa.

Finalmente, en la tabla 5, se muestra el impacto que cada variable tiene sobre la medida tradicional en la literatura para estimar los niveles de pobreza, es decir, los ingresos. En este modelo cada uno de estos elementos va a impactar de manera importante sobre los ingresos de los individuos. Los estándares de vida de los hogares aunque no significativos estadísticamente mediante sus efectos directos, parecen hacerlo de manera indirecta, lo cual es validado por el remuestreo de bootstrapping. Como se mencionó, los estándares de vida impactan sobre las condiciones de salud de los individuos, que a su vez influyen sobre el rendimiento de los logros educativos y la productividad de los trabajadores, lo cual en suma se reflejará en las remuneraciones de los trabajadores. Esto se observa en los resultados, tanto la salud como la educación tienen un fuerte impacto directo y significativo, que se amplifica al observar sus efectos indirectos. Por otra parte, en las economías modernas el papel jugado por las tecnologías para aumentar la productividad de los trabajadores es fundamental, así se evidencia en los resultados.

Una vez estimado el modelo es posible encontrar las ponderaciones de los indicadores que serán utilizados en la ecuación 1. Esto se logra primero tomando como referencia el bloque de ingresos de los individuos. Al final, este bloque representa los umbrales de pobreza, medidos a través de ingresos, y como este nivel se ve afectado por los conceptos relacionados con él. En este orden de ideas, se computa el aporte que cada uno de los conceptos hace al bloque de ingresos en la varianza. Para calcular dicha contribución, primero se toman como referencia los coeficientes de los efectos totales de cada variable y se multiplican por la correlación entre estas y el bloque de ingresos, dando como resultado la contribución a la varianza. Posteriormente, este es relativizado con respecto al coeficiente de determinación del bloque para obtener la contribución porcentual de cada variable dentro del bloque de ingresos, es decir:

$$
\text { Contri\% }=\left[\frac{\beta_{p q} \times \operatorname{corr}\left(X_{q}, \text { ingre }\right)}{R^{2}}\right]
$$

Luego, esta contribución es multiplicada por el cociente entre el peso que cada indicador tiene en el bloque al cual pertenece, con este paso finalmente se logran las ponderaciones para estimar la ecuación 1:

$$
w_{j}=\left[\frac{P e s o_{p q}}{\sum_{p} p e s o_{p q}}\right] \text { (contri\%) }
$$

La tabla 6 reporta estas estimaciones. La salud y la educación son los componentes que más aportan al índice de pobreza multidimensional. La teoría y la literatura empírica sobre los impactos de la educación y la salud señalan a estas dos variables como las más importantes para la reducción de la pobreza. A nivel de los hogares, la productividad de los individuos y el nivel de ingresos son resultados que se realizan en la medida en que estas dos variables alcanzan niveles más altos. De la misma manera, a nivel de los países, una fuerza laboral más sana y educada construirá un bloque para una base de conocimiento con un mayor crecimiento económico. Los estudios sugieren que las inversiones en capital humano son la precondición para que los países en desarrollo absorban nuevas 
tecnologías y mejoren sus productividades, lo que se refleja en ingresos más altos y mejores resultados económicos. Varios estudios han dado evidencia sobre la relación entre estas tres variables (Auster, Leveson y Sarachek, 1969; Friedman, 2002; Fuchs, 1980; Lee, 1982; Leigh, 1981). Este documento no solo va en línea con esta literatura, sino que además da estimaciones de la importancia relativa de estas variables en relación con otros factores en la contribución de la pobreza.

Tabla 6

Contribuciones para explicar la varianza - Chile

\begin{tabular}{lcccc}
\hline & Coeficiente & Correlación & Contri. Var & Contri. \% \\
\hline CONDI & 0,334 & 0,151 & 5,0 & 6,3 \\
\multirow{2}{*}{ SALUD } & 0,870 & 0,399 & 32,9 & 41,2 \\
\multirow{2}{*}{ EDU } & 0,838 & 0,378 & 33,4 & 41,9 \\
\multirow{2}{*}{ TECNO } & 0,412 & 0,206 & 8,5 & 10,6 \\
\hline
\end{tabular}

Nota. Las contribuciones se estiman con base en el producto del coeficiente total y la correlación entre conceptos en relación con el $R^{2}$. Elaboración propia con base en Indicadores para Chile, por Casen, 2009.

Una vez que son calculadas las ponderaciones, es posible estimar el índice de pobreza multidimensional. Como se mencionó, para la estimación de IPM, es necesario primero calcular el índice de recuento multidimensional, mediante la ecuación (2). La figura 1 muestra los resultados de este índice. Este índice nos señala que, en promedio, el 15,9\% de la población chilena es pobre multidimensionalmente. Al mismo tiempo, que las zonas rurales presentan un mayor nivel de pobreza, $17,8 \%$ frente al $14,4 \%$ de los regiones urbanas. En cuanto al nivel nacional los resultados encontrados por este estudio están muy cercanos a las estimaciones realizadas por otros estudios, basadas en otras metodologías, particularmente la del observatorio social del Ministerio de Desarrollo de Chile (2013). En la figura 1, también se incluye el IPM estimado a través de la ecuación 4 , el cual representa la participación de las personas que son pobres multidimensionalmente ajustadas por la intensidad de las privaciones sufridas. Los resultados revelan que en promedio a nivel nacional el 7,4\% son pobres multidimensionalmente una vez ajustada la medida. A nivel urbano este valor es de $6,8 \%$, mientras que es de $9,0 \%$ para las áreas rurales.

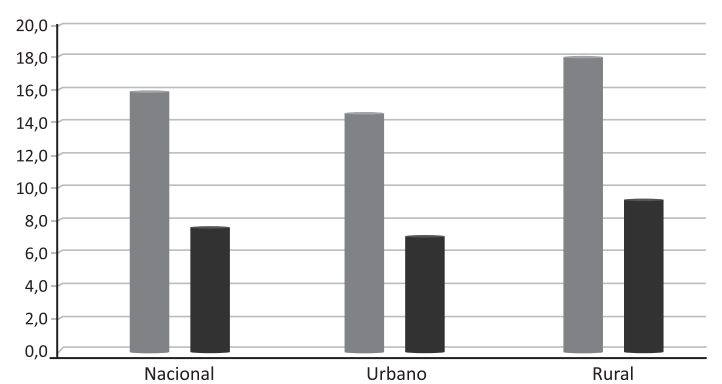

Índice de recuento multidimensional

O Índice de pobreza multidimensional

Figura 1. Índices de pobreza para Chile - 2009. Índice de recuento multidimensional (H) e índice de pobreza multidimensional (IPM) estimados de acuerdo a las ecuaciones 2 y 4 respectivamente con base en Casen (2009) del Ministerio de Planificación.

El índice presentado contiene una serie de información condensada en una sola medida, la cual es posible descomponer en varias características (para detalles del cálculo, vea Alkire y Foster, 2011). En este documento se ha querido hacer tal descomposición por regiones, para observar donde se focalizan las más atrasadas en términos de pobreza, las figuras 2 y 3 muestran estos resultados. La evidencia presenta que las regiones con niveles más pobres de país, Bío Bío (21,2\%), Rios (19,0\%), Arauquita (17,9\%), Lagos (16,7\%), entre otras, jalonan la media nacional de pobreza. Entre tanto, regiones como Magallanes $(7,3 \%)$, Altafagasta $(8,8 \%)$ y la región Metropolitana $(9,1 \%)$ presentan grandes diferencias respecto a las más pobres. Muchas de estas diferencias son explicadas por las privaciones en educación y salud de los individuos de las regiones más atrasadas. Las zonas con mejores indicadores, tanto del índice de recuento multidimensional como del IPM, presentan también los mejores indicadores de desarrollo, y en particular, los dos catalizadores encontrados en este estudio: salud y educación. En efecto, en estas regiones, sobre todo en las regiones metropolitanas, es donde se encuentra el mayor número de escuelas, universidades, centros médicos, etc.

Esta infraestructura no permite permear o extender los beneficios a otras regiones del país de manera satisfactoria. Los motivos provienen de varias fuentes, entre ellos, la distancia, las diferencias culturales, etc. Esto constituye un problema de política pública que debe ser analizado con cuidado para mejorar los indicadores de pobreza y privación de las regiones más atrasadas. 


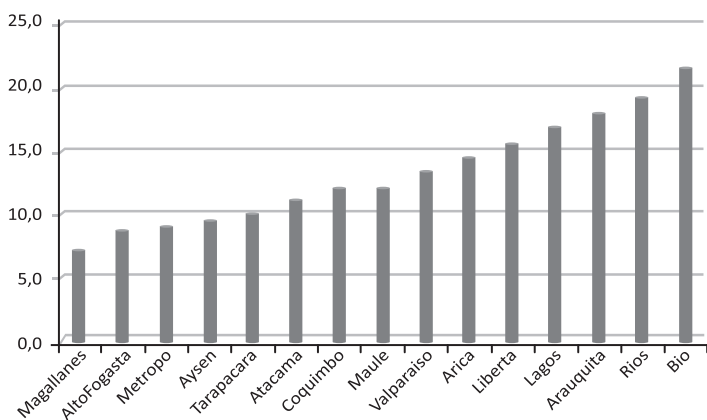

Figura 2. Índice de recuento multidimensional por regiones - 2009. Índice de recuento multidimensional $(\mathrm{H})$ estimado de acuerdo a la ecuación 2 con base en Casen (2009) del Ministerio de Planificación.

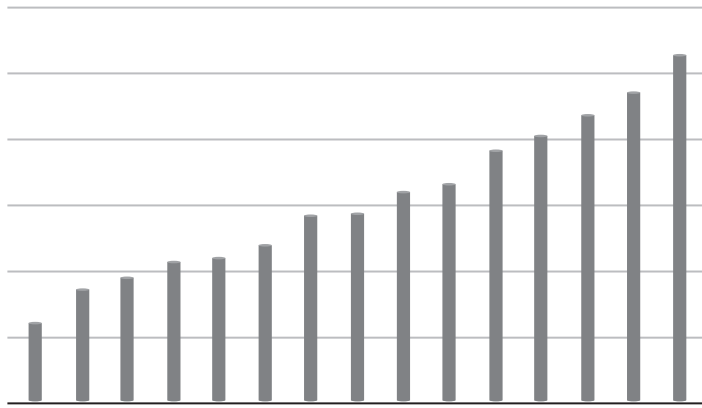

Figura 3. Índice de pobreza multidimensional por regiones, 2009. Índice de pobreza multidimensional (IPM) estimado de acuerdo con la ecuación 4 con base en Casen (2009) del Ministerio de Planificación.

\section{Pobreza multidimensional para Colombia}

La metodología del cálculo de la pobreza multidimensional expuesto anteriormente es ahora aplicada a otro país latinoamericano, con unas características diferentes a las de Chile. Colombia es un país considerado por los indicadores del Banco Mundial como uno de ingreso medio bajo con grandes contrastes en su rendimiento. Por un lado, presenta grandes problemas sociales y políticos, entre los que sobresale el conflicto armado y la delincuencia común, los altos índices de corrupción y los bajos índices en términos de confianza en algunas instituciones, como el Congreso de la República, por parte de los ciudadanos. Por otro, presenta una de las democracias más sólidas del continente y con estable y relativo crecimiento económico. Esto permite hacer un contraste de los indicadores de pobreza entre estos dos países con diferencias tan marcadas.

En primer lugar, la tabla 7 muestra las estimaciones de los índices de Rho de Dillon-Goldstein y valores propios de la matriz de varianza-covarianza para evaluar unidimensionalidad para el caso de Colombia. $\mathrm{Al}$ igual que Chile, se comprueba que los modelos externos o de medida son todos unidimensionales, lo que señala que los indicadores que se emplean caracterizan bien a los conceptos.

Tabla 7

Unidimensionalidad de los indicadores de pobreza - Colombia

\begin{tabular}{lccccc}
\hline & Modo & VM & Rho. DG & 1er. Igo & 2do. Igo \\
\hline $\begin{array}{l}\text { Estándar } \\
\text { de vida }\end{array}$ & $\mathrm{A}$ & 1 & 1000 & 1,00 & 0,00 \\
Salud & $\mathrm{A}$ & 2 & 0,783 & 1,27 & 0,73 \\
Educación & $\mathrm{A}$ & 3 & 0,741 & 1,41 & 0,59 \\
TIC & $\mathrm{A}$ & 2 & 0,819 & 1,83 & 0,47 \\
Ingresos & $\mathrm{A}$ & 1 & 1000 & 1,00 & 0,00 \\
\hline
\end{tabular}

Nota. Estimaciones basadas en un modelo reflectivo, esquema centroide y variables estandarizadas. Elaboración propia con base en indicadores de la ENCV del DANE.

Por otro lado, las cargas de los diferentes indicadores muestran un comportamiento aceptable dentro de los diferentes bloques. Solamente los indicadores de estatus de salud y la educación de los padres no pasan la prueba estricta de $\lambda>0.7$, pero son valores superiores a 0.6 , con lo cual empíricamente se consideran relevantes para explicar los bloques que tratan de medir (ver tabla 8).

$\mathrm{Al}$ igual que antes, un test adicional para validar los resultados de Colombia se encuentra en la evaluación discriminante. En la tabla 9 se encuentra que los diferentes indicadores miden bien sus conceptos puesto que ninguno de ellos da una carga más alta en ningún otro bloque al que trata de medir.

Una vez validados los modelos de medida, se analizan los resultados del sistema presentado en las ecuaciones 5-8 para el caso de Colombia. En términos generales, los resultados muestran comportamientos similares a los encontrados en Chile. En primer lugar, en promedio, la salud tiene efectos positivos sobre los estándares de vida, los logros educativos y las remuneraciones salariales de los individuos. De la misma manera, en promedio, las mejores condiciones en los estándares de vida de los hogares mejoran positivamente los ingresos y la educación de los trabajadores. 
Tabla 8

Pesos y cargas del modelo de pobreza - Colombia

\begin{tabular}{cccccc}
\hline & VM & Bloque & Pesos & Cargas & Comunalidad \\
\hline 1 & SERVI & $\begin{array}{c}\text { Estándar } \\
\text { de vida }\end{array}$ & 1000 & 1000 & 1000 \\
2 & AFI & Salud & 0,739 & 0,759 & 0,676 \\
3 & SALU & Salud & 0,652 & 0,673 & 0,653 \\
4 & APE & Educación & 0,887 & 0,908 & 0,825 \\
5 & ANA & Educación & 0,591 & 0,749 & 0,549 \\
6 & EDUPA & Educación & 0,479 & 0,603 & 0,501 \\
7 & CELU & TIC & 0,630 & 0,735 & 0,712 \\
8 & PC & TIC & 0,721 & 0,852 & 0,916 \\
9 & Y & Ingresos & 1000 & 1000 & 0,915 \\
\hline
\end{tabular}

Nota. Estimaciones basadas en un modelo reflectivo, esquema centroide y variables estandarizadas. Elaboración propia con base en ENCV del DANE.

Al mismo tiempo, esta última variable impacta de manera positiva y fuertemente sobre la capacidad que tienen los individuos de operar la tecnología y la información así como los salarios. Por último, cada una de estas variables tiene un fuerte y significativo impacto para aumentar la productividad que se refleja en los salarios de los trabajadores, no solo por sus efectos directos, sino además por los que se producen entre las retroalimentaciones del sistema. En términos relativos, el modelo señala que los factores relacionados directamente sobre la formación de capital humano (salud y educación) son los que más impactan sobre los ingresos de los trabajadores.
Esta última afirmación es confirmada también al calcular la contribución que cada variable aporta al bloque de los ingresos de los trabajadores. La salud aporta en promedio el 34,5\% del total de la varianza, mientras la educación lo hace con el 45,6\%, es decir, que solo estas dos variables explican más del $80 \%$ de las variaciones de las remuneraciones salariales.

Esto ratifica los resultados encontrados para Chile, en los que estas variables, esenciales para la formación del capital humano son las que van a impactar de manera significativa en las reducciones de la pobreza y, por lo tanto, donde se deberían concentrar los mayores esfuerzos en la política pública.

Por otro lado, las diferencias entre Colombia y Chile son significativas en términos de indicadores de pobreza. Mientras, como se mencionó, la pobreza en Chile alcanza niveles del 16\%, medido a través de los índices de recuento multidimensional (н) y pobreza multidimensional (IPM); en Colombia, esta cifra se duplica al alcanzar en el 2012 un valor de 35,9\% a nivel nacional medida a través de $\mathrm{H}$ y del 17,4\% a través de IPM (figuras 3 y 4). Las diferencias entre las zonas urbanas y rurales son más extremas en este país, miradas a través del índice de recuento multidimensional. Mientras que la pobreza en las zonas urbanas alcanza el $36,4 \%$, en las zonas rurales esta cifra es de casi el $60 \%$. Cuando este indicador es ajustado -el índice de pobreza multidimensional-, las zonas rurales presentan ratios que van más allá de 1,6 puntos por encima de las zonas urbanas. Lamentablemente el conflicto armado, las reformas estructurales de los años noventa, la volatilidad de los precios de los

Tabla 9

Correlación por bloques para el modelo de pobreza - Colombia

\begin{tabular}{lccccccc}
\hline & Nombre & Bloque & Condi. & Salud & Educa. & TIC & Ingre. \\
\hline 1 & SERVI & Estándar de vida & 1000 & 0,499 & 0,545 & 0,347 & 0,528 \\
2 & AFI & Salud & 0,463 & 0,961 & 0,663 & 0,473 & 0,634 \\
3 & SALU & Salud & 0,480 & 0,829 & 0,622 & 0,451 & 0,576 \\
4 & APE & Educación & 0,633 & 0,731 & 0,931 & 0,643 & 0,621 \\
5 & ANA & Educación & 0,383 & 0,548 & 0,852 & 0,492 & 0,385 \\
6 & EDUPA & Educación & 0,510 & 0,487 & 0,788 & 0,274 & 0,364 \\
7 & CELU & TIC & 0,318 & 0,389 & 0,397 & 0,390 & 0,383 \\
8 & PC & TIC & 0,493 & 0,204 & 0,536 & 0,123 & 0,322 \\
9 & Y & Ingresos & 0,667 & 0,607 & 0,655 & 0,493 & 1000 \\
\hline
\end{tabular}

Nota. Estimaciones basadas en un modelo reflectivo, esquema centroide y variables estandarizadas. Elaboración propia con base en ENCV del DANE. 
Tabla 10

Modelo PLS-PM base - Colombia

\begin{tabular}{|c|c|c|c|c|}
\hline Bloques & Coeficiente & Directos & Indirectos & Totales \\
\hline \multirow[t]{2}{*}{ Salud $\rightarrow$ Están. } & $0,499^{* * *}$ & 0,499 & & $0,499 \dagger$ \\
\hline & $(0,047)$ & & & $(0,015)$ \\
\hline Coeficiente de determinación, $R^{2}$ & 0,598 & & & \\
\hline \multirow[t]{2}{*}{ Están $\rightarrow$ Edu } & $0,126^{* * *}$ & 0,126 & & $0,126 \dagger$ \\
\hline & $(0,004)$ & & & $(0,003)$ \\
\hline \multirow[t]{2}{*}{ Salud $\rightarrow E d u$} & $0,327^{* * *}$ & 0,327 & 0,114 & $0,441 \dagger$ \\
\hline & $(0,004)$ & & & $(0,013)$ \\
\hline Coeficiente de determinación, $R^{2}$ & 0,739 & & & \\
\hline \multirow[t]{2}{*}{$E d u \rightarrow$ Tecno } & $0,215^{* * *}$ & 0,215 & & $0,215 \dagger$ \\
\hline & $(0,005)$ & & & $(0,017)$ \\
\hline Coeficiente de determinación, $R^{2}$ & 0,746 & & & \\
\hline \multirow[t]{2}{*}{ Están. $\rightarrow$ Ingre } & $0,163^{* * *}$ & 0,163 & 0,331 & $0,494 \dagger$ \\
\hline & $(0,023)$ & & & $(0,005)$ \\
\hline \multirow[t]{2}{*}{ Salud $\rightarrow$ Ingre } & $0,659^{\star * *}$ & 0,659 & 0,105 & $0,764 \dagger$ \\
\hline & $(0,026)$ & & & $(0,003)$ \\
\hline \multirow[t]{2}{*}{ Edu $\rightarrow$ Ingre } & $0,667^{* * *}$ & 0,667 & 0,279 & $0,946 \dagger$ \\
\hline & $(0,029)$ & & & $(0,006)$ \\
\hline \multirow[t]{2}{*}{ Tecno $\rightarrow$ Ingre } & $0,318^{\star * *}$ & 0,318 & & $0,318 \dagger$ \\
\hline & $(0,039)$ & & & $(0,029)$ \\
\hline Coeficiente de determinación, $R^{2}$ & 0,838 & & & \\
\hline GoF & 0,757 & & & \\
\hline
\end{tabular}

Nota. Modelo estimado para variables latentes estandarizadas. Errores estándar en paréntesis. ${ }^{* *} \mathrm{p}<0,01 .{ }^{\dagger}$ La validación de la significancia de los coeficientes de los efectos totales está basada en intervalos de confianza al 95\% con el método de remuestreo por bootstrapping. Elaboración propia con base en ENCV del DANE.

commodities y el abandono del estado en las zonas rurales pueden explicar mucho de las marcadas diferencias entre estas dos zonas.

Ahora, son evidentes las marcadas diferencias entre estos dos países: mientras Chile presenta

Tabla 11

Contribuciones para explicar la varianza explicada - Colombia

\begin{tabular}{lcccc}
\hline & Coeficiente & Correlación & $\begin{array}{c}\text { Contri. } \\
\text { Var }\end{array}$ & $\begin{array}{c}\text { Contri. } \\
\%\end{array}$ \\
\hline CONDI & 0,494 & 0,251 & 12,4 & 14,8 \\
SALUD & 0,764 & 0,379 & 29,0 & 34,5 \\
EDU & 0,946 & 0,404 & 38,2 & 45,6 \\
TECNO & 0,318 & 0,134 & 4,26 & 5,08 \\
\hline
\end{tabular}

Nota. Las contribuciones se estiman con base en el producto del coeficiente total y la correlación entre conceptos con relación al $R^{2}$. Elaboración propia en base a ENCV del DANE. índices de pobreza relativamente pequeños, para Colombia estos se tornan preocupantes. Los fenómenos que expliquen dichas diferencias escapan al objetivo de este artículo, pero se convierte en una partida para explorar los determinantes que inciden en estos resultados. De todos modos, se puede afirmar que las políticas públicas y económicas tomadas por ambos países pueden haber contribuido de manera significativa en este proceso. Aunque en teoría ambos países han adelantado procesos de liberación de los mercados y reformas institucionales, en la práctica, los resultados parecen hacerse más palpables para el caso de Chile. Posiblemente, el contexto colombiano, mucho más complejo por el conflicto armado, no permite extrapolar los beneficios del crecimiento económico. En efecto, otros estudios han mostrado que mientras Chile ha venido reduciendo vertiginosamente sus indicadores a través del tiempo, dicha reducción ha sido modesta en el caso de Colombia. 


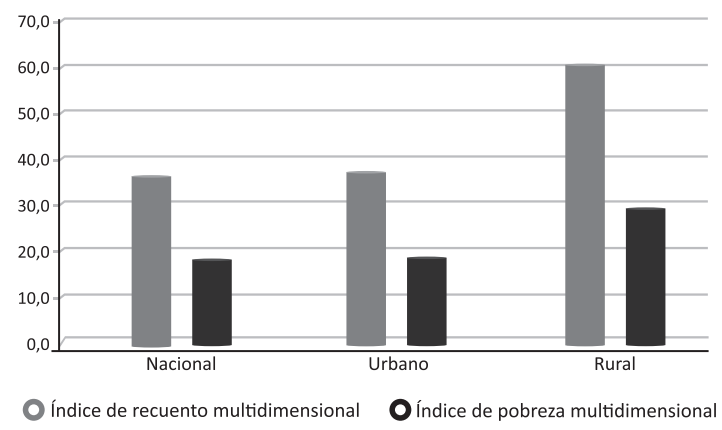

Figura 4. Índices de pobreza para Colombia - 2012. Índice de recuento multidimensional (H) e índice de pobreza multidimensional (IPM) estimados de acuerdo a las ecuaciones (2) y (4) respectivamente con base en GEIH 2012 del DANE.

Por otro lado, por regiones, el índice de recuento multidimensional muestra que las zonas más pobres del país se encuentran en Chocó, donde la pobreza alcanza la sorprendente cifra del $71,4 \%$, Guajira $(65,2 \%)$, Córdoba $(63,2 \%)$, Cauca $(61,3)$, Nariño $(54,9 \%)$, entre otras (figura 5 y 6). Estos resultados son llamativamente preocupantes no solo porque como en el caso de Chocó que de cada 100 personas 70 son pobres multidimensionalmente, sino además porque las diferencias son demasiado marcadas con respecto a otras regiones: los niveles de pobreza en zonas como Bogotá son del 12,2\%, en Cundinamarca del 21,8\% y en Antioquia del 24,5\%. Los datos para estas últimas regiones no están lejanos de los observados por las zonas más ricas de Chile, lo cual demuestra que el mayor reto para Colombia se encuentra en reducir estos indicadores para las zonas más deprimidas del país. El centralismo del país es evidente, Bogotá concentra la mayoría del poder económico, político y social del país, situación que se refleja en resultados económicos importantes, entre ellos el PIB, la recaudación tributaria, etc. Esto es importante porque algunos estudios han encontrado que algunas zonas geográficas que caen en trampas de pobreza incluso han estar ubicadas en proximidades a zonas más ricas, situación que ha sido señalada:

Los niveles de persistencia en la pobreza a menudo están asociados a los "efectos de vecindario". Estos efectos crean trampas de pobreza que no le permiten a las zonas rezagadas avanzar hacia una senda de desarrollo económico sostenido. En las regiones de un país también pueden operar este tipo de mecanismos. Esa es una de las razones por las cuales las desigual- dades territoriales se vuelven persistentes, convirtiéndose en un equilibrio perverso. En Colombia las desigualdades regionales se han mantenido y se han vuelto persistentes (Galvis y Roca, 2010, p. 1).

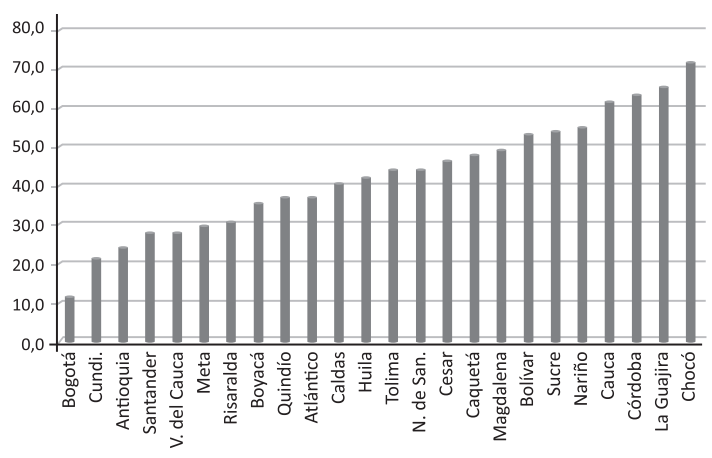

Figura 5. Índice de recuento multidimensional por regiones - 2012 Índice de recuento multidimensional $(\mathrm{H})$ estimado de acuerdo con la ecuación 2 con base en GEIH 2012 del DANE.

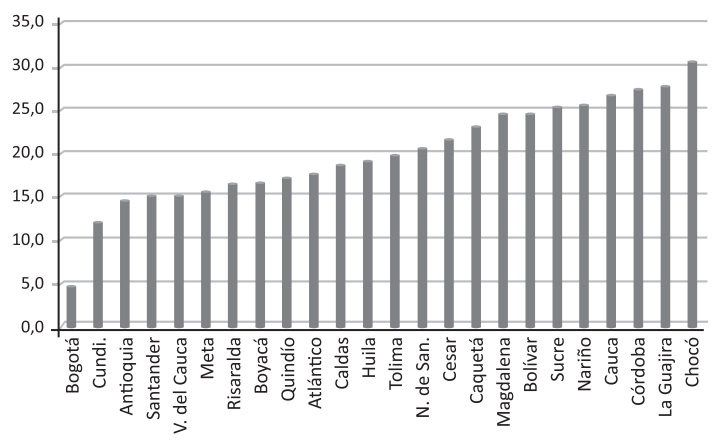

Figura 6. Índice de pobreza multidimensional por regiones - 2009. Índice de pobreza multidimensional (IPM) estimado de acuerdo con la ecuación 4 con base en GEIH 2012 del DANE.

En el 2006, el Gobierno nacional mediante un documento Conpes - Consejo de política económica y social- estableció como políticas la lucha contra la pobreza extrema y los auxilios necesarios para aliviar la pobreza, mediante programas sociales y la ayuda a los desplazados. Sin embargo, es evidente que estos resultados no han logrado su objetivo, pues las diferencias son muy marcadas entre las zonas del país.

\section{Conclusiones}

En América Latina se ha medido la pobreza a través de diversos indicadores. Muchos de ellos toman como base diferentes metodologías dentro de las 
cuales el ingreso resulta ser el indicador más importante. También se tienen en cuenta indicadores cualitativos como las Necesidades Básicas Insatisfechas (NBI) y con base en ello se construyen "líneas" o "umbrales" de pobreza.

La presente investigación ofrece una metodología novedosa para el cálculo de la pobreza y toma como variable el enfoque multidimensional en el cual se tienen en cuenta indicadores objetivos y subjetivos. Mediante esta metodología se crearon ponderadores que miden el peso de cada dimensión al momento de calcular la pobreza multidimensional, los cuales se establecieron con base en elementos estadísticos y aquellos obtenidos de la literatura reciente al respecto.

El aporte fundamental de este documento es que a diferencia de otros estudios que emplean enfoques multidimensionales, se consigue estimar de manera sistemática las ponderaciones que cada dimensión aporta a la construcción del indicador de pobreza. Esto no es un tema menor, pues la literatura alrededor de pobreza multidimensional asigna de manera arbitraria dichas ponderaciones en la formación del IPM. La importancia relativa de cada factor no impacta de manera simétrica las privaciones de los hogares $\mathrm{y}$, por lo tanto, es incorrecto asignarles un peso similar.

Un segundo aporte de este estudio es que obtiene las ponderaciones mediante una novedosa metodología, la cual ha sido poco explorada en áreas de economía. Dicha metodología permite obtener los parámetros mediante un enfoque de equilibrio, construyendo un sistema de ecuaciones que interrelacionan conceptos atados a la noción de pobreza. Las relaciones de causalidad entre estas variables se basan en la literatura alrededor de la pobreza, formando de esta manera el índice de pobreza multidimensional. Una ventaja de este método es que permite obtener no solo los efectos directos que se producen entre las relaciones de las variables, sino también los indirectos, aquellos que se dan entre las retroalimentaciones del sistema. Adicionalmente, el modelo permite obtener con precisión la importancia relativa de cada variable en la formación del concepto de pobreza, evitando, de esta manera, subjetividades presentes en otras investigaciones y, por lo tanto, sesgos en las estimaciones.

Para el estudio se tomó información proveniente de la Encuesta de Caracterización Socioeconómica (Casen), para el caso de Chile, 2009, yla Gran Encuesta Integrada de Hogares (GEIH) para Colombia, 2012.

El análisis de la evidencia empírica mostró que Chile cuenta con una menor concentración del ingreso que Colombia. Mientras que en Chile el $1 \%$ más rico de la población encuestada tiene un ingreso 36 veces mayor que el $5 \%$ de menores ingresos, en Colombia esta misma proporción es de 106 veces.

En cuanto a los niveles de educación, aunque los dos países muestran tasas de alfabetismo relativamente similares, en Chile los niveles de escolaridad y capacitación de la población superan a los de los colombianos. Aunque no hace parte del ámbito de la presente investigación, este resultado es relevante para posteriores estudios en los que se analicen las diferencias en la productividad.

Aunque los resultados de las encuestas no arrojan evidencia en torno a aspectos cualitativos de salud, la cobertura en ambos países resulta muy similar, destacándose el régimen subsidiado como el sistema al que se encuentra afiliada la mayoría de la población. Los indicadores de calidad de vida muestran que en Chile existe un mayor cubrimiento del acceso a internet y a la telefonía móvil celular. En cuanto a salubridad e higiene, es preocupante observar que aún existe un porcentaje de los hogares encuestados en Colombia (aunque bajo) que no cuenta con sistema adecuado de eliminación de excretas (no tiene sanitario).

La evidencia presentada en este documento señala que los componentes más importantes para la reducción de la pobreza los constituyen los logros educativos y el estatus en salud. Estos elementos son fundamentales para la política pública, pues como señala la evidencia no solamente tienen un fuerte y significativo impacto en la formación del IPM, sino que además, entre ellos dos se explica más del $80 \%$ de la varianza. La salud impacta de manera directa e indirecta sobre muchos factores que redundan en las privaciones de los hogares, particularmente en los logros educativos y en la productividad de los trabajadores, lo que al final incide sobre las remuneraciones de estos. Además constituye la plataforma en la cual la educación es mejor aprovechada. Por su parte, la educación es el mecanismo por el cual se reducen las desigualdades al aumentar el capital humano, impactando directamente sobre las productividades de los trabajadores, así como aumentando las capacidades de estos para generar y absorber nuevas tecnologías, lo que incide sobre sus posibilidades para encontrar trabajo e incrementar su poder de negociación. En este estudio, se demuestra que la educación porque incrementa el aprovechamiento de las tecnologías de la información y la comunicación aumentando aún más sus efectos sobre las remuneraciones de los trabajadores. 
La aplicación empírica de la metodología propuesta permite dilucidar las grandes diferencias entre dos países latinoamericanos. Por un lado, los resultados muestran que en el caso de Chile, la pobreza alcanza niveles del $16 \%$ a nivel nacional, 20 puntos por debajo de los niveles presentados por Colombia. Cuando el índice es ajustado por privaciones Chile registra un IPM de 7,4\% mientras Colombia un $17,4 \%$. Esto revela los marcados contrastes entre estos dos países a pesar de estar en regiones relativamente cercanas. Las reformas estructurales hechas por Chile parecen estar teniendo un mejor recibimiento que en el caso de Colombia. Chile ha mostrado una tendencia de mejora desde los años 70 ubicándose en la actualidad como el mejor país del continente en términos de bienestar e ingreso per cápita. Sin embargo, los resultados encontrados en este estudio señalan que aún hay mucho por mejorar pues en comparación con países de mayor desarrollo, el IPM es aún muy alto. En el caso de Colombia, las preocupaciones son alarmantes, los niveles de pobreza son muy altos incluso comparado con países de la región. Las diferencias entre este país y Chile pueden deberse al contexto particular de Colombia, el cual se encuentra en un conflicto armado que le impide canalizar con mayor efectividad los diseños de política pública.

Por otro lado, la pobreza entre zonas urbanas y rurales es también más marcada en el caso de Colombia. Mientras que, para Chile, las zonas urbanas muestran un índice de pobreza de alrededor del 14\% y, para zonas rurales, del 18\%; en Colombia, estos son del $36 \%$ y $60 \%$ respectivamente. En la actualidad, la homogeneidad entre zonas urbanas y rurales vivida en Chile contrasta con la inequidad de Colombia. El abandono del estado, la apertura económica, el conflicto armado y el narcotráfico pueden explicar la concentración de la pobreza en las zonas rurales colombianas. La apertura económica de los años noventa parece haber impactado negativamente a los agricultores colombianos que no han recibido ayudas del gobierno nacional para mitigar los efectos de la competitividad. Este sector ha necesitado de reformas estructurales que no se han implementado de tal manera que le permitan tecnificarse y hacerse más competitivos para hacer frente a la volatilidad de los commodities, a la tasa de cambio y, en general, a la competencia externa. Los agricultores colombianos han sufrido las consecuencias de la guerra, disminuyendo su productividad y los recursos disponibles, situación que impacta negativamente sobre sus ingresos.
Por regiones, los resultados muestran como las áreas metropolitanas concentran los menores índices de pobreza. En el caso de Chile, áreas como Bío Bío, Ríos, Arauquita, etc., reportan los mayores índices de pobreza con niveles de más de 12 puntos por encima de regiones como Magallanes, Altafagosta y el área Metropolitana. Esto demuestra para este país la necesidad de profundizar las políticas de reducción de la pobreza, particularmente a través de educación y salud, con el ánimo de reducir las desigualdades entre regiones. Para el caso de Colombia, nuevamente las cosas se tornan mucho más preocupantes. Mientras que Bogotá reporta índices cercanos a las áreas metropolitanas de Chile, áreas como Chocó, Guajira, Córdoba, etc., presentan índices de pobreza de más del 60\%. Estas regiones no parecen aprovecharse ni del crecimiento económico ni de las políticas públicas ni de la cercanía de regiones adyacentes. La concentración de la riqueza y el poder político y económico en el centro del país no permite transferir estos beneficios a las periferias, las cuales generalmente son las que sufren las consecuencias de la guerra.

\section{Referencias}

Angulo, R., Díaz, Y. y Pardo, R. (2011). Índice de Pobreza Multidimensional para Colombia (IPM-Colombia) 1997-2010. Archivos de Economía, documento 382, Bogotá: Departamento Nacional de Planeación.

Auster, R., Leveson, I. y Sarachek, D., (1969). The production of health: an exploratory study. Journal of Human Resources 4, 411-436. http://dx.doi.org/10.2307/145166

Alkire, S., y Foster, J.E. (2011). Counting and Multidimensional Poverty Measurement. Journal of Public Economics. 95(7-8). 476-487. http://dx.doi.org/10.1016/j. jpubeco.2010.11.006

Alkire, S. y Santos, M. E. (2010). Training Material for Producing National Human Development Reports. Disponible en www.ophi. org.uk/wp-content/uploads/ OPHI-RP-31a. pdf.pdf?cda6c1

Atkinson, A. B. (1983). The Economics of Inequality (2nd ed.). Oxford: Clarendon Press.

Atkinson, A. B. (1987). On the Measurement of Poverty. Econometrica. 4, 749-764. http://dx.doi.org/10.2307/ 1911028

Friedman, H. S. (2002). Health Psychology (2nd ed.). New Jersey: Pearson Education.

Fuchs, V. (1980). Time preference and health: an exploratory study. (NBER working paper N. ${ }^{\circ} 539$ ). New York: National Bureau of Economic Research. 
Galvis, L. y Roca, A. (2010). Persistencia de las desigualdades regionales en Colombia: Un análisis especial. (Documentos de Trabajo sobre Economía Regional). Bogotá: Banco de la República.

Gasparini, L., Gutiérrez, F. y Tornarolli, L. (2007). Growth and income poverty in Latin America and the Caribbean: evidence from household surveys. Review of Income and Wealth, 53(2), 209-245. http://dx.doi.org/10.1111/j.1475-4991.2007.00231.x

Gefen, D., Straub, D. W., y Boudreau, M. C. (2000). Structural equation modeling and regression: guidelines for research practice. Communications of the Association for Information Systems, 4(7). 178.

Lasso, F. J. (2008). Impacto de los cambios de precios relativos en pobreza y desigualdad en Colombia: 1998 2007. Ensayos sobre Política Económica, N. ${ }^{\circ} 57$. Bogotá: Banco de la República.

Lee, L. F. (1982). Health and wage: a simultaneous equation model with multiple discrete indicators. International Economic Review, 23, 199-222. http://dx.doi. org/10.2307/2526472

Leigh, J. P. (1981). Hazardous occupations, illness and schooling. Economics of Education Review, 2, 381-388. http://dx.doi.org/10.1016/0272-7757(81)90006-6

Lohmoller, J. B. (1989). Latent variable path modeling with partial least squares. Heidelberg: Physica.

López-Aranguren, E. (2005). Problemas sociales. Desigualdad, pobreza, exclusión social. Madrid: Biblioteca Nueva.
López, H. y Núñez, J. (2007). Pobreza y desigualdad en Colombia: diagnóstico y estrategias. Misión para el diseño de una estrategia para la reducción de la pobreza y la desigualdad - MERPD. Bogotá: Departamento Nacional de Planeación.

Martínez-Álvarez, J. A. (2003). Economía de la pobreza. Madrid: UNED Ediciones.

Ministerio de Desarrollo de Chile. (2013). Incidencia de la pobreza a nivel comunal, según metodología de estimación para áreas pequeñas. Chile 2009 y 2011. Serie Informes Comunales, N. ${ }^{\circ} 1$.

Nuñez, J., y Ramírez J. C. (2002). Determinantes de la pobreza en Colombia. Años recientes. Serie estudios y perspectivas. Bogotá: CEPAL.

Pérez, G. (2005). Dimensión espacial de la pobreza en Colombia. Ensayos sobre Política Económica, N. ${ }^{\circ} 48$. Bogotá: Banco de la República

Poza, C. (2008). Pobreza multidimensional: el caso específico español a través del panel de hogares de la Unión Europea. (Tesis doctoral, Universidad Complutense de Madrid, Madrid).

Saboor, A., Khan, A. U., Hussain, A. y Mahmood, K. (2015). Multidimensional Poverty in Pakistan: Regional Variations and Temporal Shifts. Quarterly Review of Economics and Finance, 56, 57-67. http://dx.doi.org/10.1016/j.qref.2015.02.007.

Ureña, C. (1999). Contraste entre medidas objetivas y subjetivas de pobreza. Reunión del Grupo de Río. Lisboa: INE. Recuperado de http://www.ibge.gov.br/poverty/ pdf/carmen_unrena_espana.pdf 